\title{
Spin and charge ordering in self-doped Mott insulators
}

\author{
T. Mizokawa, D. I. Khomskii, and G. A. Sawatzky \\ Solid State Physics Laboratory, Materials Science Centre, University of Groningen, Nijenborgh 4, 9747 AG Groningen, The \\ Netherlands
}

(November 21, 2018)

\begin{abstract}
We have investigated possible spin and charge ordered states in $3 d$ transition-metal oxides with small or negative charge-transfer energy, which can be regarded as self-doped Mott insulators, using Hartree-Fock calculations on $d$ - $p$-type lattice models. It was found that an antiferromagnetic state with charge ordering in oxygen $2 p$ orbitals is favored for relatively large charge-transfer energy and may be relevant for $\mathrm{PrNiO}_{3}$ and $\mathrm{NdNiO}_{3}$. On the other hand, an antiferromagnetic state with charge ordering in transition-metal $3 d$ orbitals tends to be stable for highly negative charge-transfer energy and can be stabilized by the breathing-type lattice distortion; this is probably realized in $\mathrm{YNiO}_{3}$.
\end{abstract}

The electronic structure of $3 d$ transition metal oxides is described by Zaanen-Sawatzky-Allen (ZSA) scheme [1] in which they are classified into two regimes according to the relative magnitude of the oxygen-to-metal chargetransfer energy $\Delta$ and the $d-d$ Coulomb interaction energy $U$. While the magnitude of the band gap is given by $U$ in the Mott-Hubbard regime, it is given by $\Delta$ in the charge-transfer regime $\Delta<U .3 d$ transition-metal oxides with high valence generally have very small or negative charge-transfer energy $\Delta$ and fall in a region which is not included in the ZSA scheme [2]. Actually, perovskitetype $3 d$ transition-metal oxides such as $\mathrm{LaCu}^{3+} \mathrm{O}_{3}$, [3] $\mathrm{PrNi}^{3+} \mathrm{O}_{3}$ [4] and $\mathrm{SrFe}^{4+} \mathrm{O}_{3}$ [5] have been studied by high energy spectroscopy and have been found to have very small or even negative charge-transfer energy $\Delta$.

With small or negative $\Delta$, the highest part of the oxygen $2 p$ bands can overlap with the lowest part of the upper Hubbard band constructed from the transition-metal $3 d$ orbitals so that some holes are transferred from the $3 d$ orbitals to the $2 p$ orbitals in the ground state. This state can be viewed as a self-doped state of a Mott insulator [6] such as has recently been suggested for $\mathrm{CrO}_{2}$ [7]. The properties of such system are far from clear and can be very rich. It can be a paramagnetic metal, a ferromagnetic (FM) metal, and a non-magnetic insulator similar to Kondo-insulators [b]. However, there exists another possibility which has not been explored until now: it may have charge ordering or charge density wave. It is possible that, in a self-doped state of a Mott insulator, holes in the oxygen $2 p$ orbitals undergo charge ordering just like doped Mott insulators such as $\mathrm{La}_{2-x} \mathrm{Sr}_{x} \mathrm{NiO}_{4}$ [8]. In this letter, we study this possibility using model HartreeFock (HF) calculation and show that spin and charge ordered states may appear in perovskites with negative $\Delta$. Based on the calculations, we argue that this phenomena occurs in perovskites containing $\mathrm{Fe}^{4+}\left(\mathrm{CaFeO}_{3}\right)[9]$ and $\mathrm{Ni}^{3+}\left(R \mathrm{NiO}_{3}\right.$ where $R$ is a rare earth) [10]. Specifically, we consider the latter system, properties of which, especially its strange magnetic properties remain a puzzle until now [4, 11].
We use the multi-band $d$ - $p$ model with $16 \mathrm{Ni}$ sites in which full degeneracy of the $\mathrm{Ni} 3 d$ orbitals and the oxygen $2 p$ orbitals are taken into account [12]. The Hamiltonian is given by

$$
\begin{gathered}
H=H_{p}+H_{d}+H_{p d}, \\
H_{p}=\sum_{k, l, \sigma} \epsilon_{k}^{p} p_{k, l \sigma}^{+} p_{k, l \sigma}+\sum_{k, l>l^{\prime}, \sigma} V_{k, l l^{\prime}}^{p p} p_{k, l \sigma}^{+} p_{k, l^{\prime} \sigma}+H . c . \\
H_{d}=\epsilon_{d} \sum_{i, m \sigma} d_{i, m \sigma}^{+} d_{i, m \sigma}+u \sum_{i, m} d_{i, m \uparrow}^{+} d_{i, m \uparrow} d_{i, m \downarrow}^{+} d_{i, m \downarrow} \\
+u^{\prime} \sum_{i, m \neq m^{\prime}} d_{i, m \uparrow}^{+} d_{i, m \uparrow} d_{i, m^{\prime} \downarrow}^{+} d_{i, m^{\prime} \downarrow} \\
+\left(u^{\prime}-j^{\prime}\right) \sum_{i, m>m^{\prime}, \sigma} d_{i, m \sigma}^{+} d_{i, m \sigma} d_{i, m^{\prime} \sigma}^{+} d_{i, m^{\prime} \sigma} \\
+j^{\prime} \sum_{i, m \neq m^{\prime}} d_{i, m \uparrow}^{+} d_{i, m^{\prime} \uparrow} d_{i, m \downarrow}^{+} d_{i, m^{\prime} \downarrow} \\
+j \sum_{i, m \neq m^{\prime}} d_{i, m \uparrow}^{+} d_{i, m^{\prime} \uparrow} d_{i, m^{\prime} \downarrow}^{+} d_{i, m \downarrow}, \\
H_{p d}=\sum_{k, m, l, \sigma} V_{k, l m}^{p d} d_{k, m \sigma}^{+} p_{k, l \sigma}+H . c .
\end{gathered}
$$

$d_{i, m \sigma}^{+}$are creation operators for the $3 d$ electrons at site $i . d_{k, m \sigma}^{+}$and $p_{k, l \sigma}^{+}$are creation operators for Bloch electrons with wave vector $k$ which are constructed from the $m$-th component of the $3 d$ orbitals and from the $l$-th component of the $2 p$ orbitals, respectively. The intraatomic Coulomb interaction between the $3 d$ electrons is expressed using Kanamori parameters, $u, u^{\prime}, j$ and $j^{\prime}$ [13]. The transfer integrals between the transition-metal $3 d$ and oxygen $2 p$ orbitals $V_{k, l m}^{p d}$ are given in terms of Slater-Koster parameters $(p d \sigma)$ and $(p d \pi)$. The transfer integrals between the oxygen $2 p$ orbitals $V_{k, l l^{\prime}}^{p p}$ are expressed by $(p p \sigma)$ and $(p p \pi)$. Here, the ratio $(p d \sigma) /(p d \pi)$ 
is -2.16. $(p p \sigma)$ and $(p p \pi)$ are fixed at -0.60 and 0.15 , respectively, for the undistorted lattice. When the lattice is distorted, the transfer integrals are scaled using Harrison's law. 14 The charge-transfer energy $\Delta$ is defined by $\epsilon_{d}^{0}-\epsilon_{p}+n U$, where $\epsilon_{d}^{0}$ and $\epsilon_{p}$ are the energies of the bare $3 d$ and $2 p$ orbitals and $U(=u-20 / 9 j)$ is the multipletaveraged $d-d$ Coulomb interaction. $\Delta, U$, and $(p d \sigma)$ for $\mathrm{PrNiO}_{3}$ are 1.0, 7.0, and $-1.8 \mathrm{eV}$, respectively, which are taken from the photoemission study [4].

The formally $\mathrm{Ni}^{3+}$ (low-spin $d^{7}$ ) compounds $R \mathrm{NiO}_{3}$ exhibit a metal-insulator transition as a function of temperature and the size of the $R$ ion [10]. Among them, $\mathrm{PrNiO}_{3}$ and $\mathrm{NdNiO}_{3}$ are antiferromagnetic insulators below the metal-insulator transition temperature. Neutron diffraction study of $\mathrm{PrNiO}_{3}$ and $\mathrm{NdNiO}_{3}$ has shown that the magnetic structure has a propagation vector of $(1 / 2,0,1 / 2)$ with respect to the orthorhombic unit cell or is a up-up-down-down stacking of the ferromagnetic planes along the $(1,1,1)$-direction of the pseudocubic lattice (see Fig. 1(a)) 11. In order to explain the magnetic structure, orbital ordering of the $x^{2}-y^{2}$ and $3 z^{2}-r^{2}$ orbitals has been proposed because one of the $e_{q}$ orbitals is occupied in the low-spin $d^{7}$ configuration [11]. However, previous model HF calculations have shown that the orbital ordered state of $x^{2}-y^{2} / 3 z^{2}-r^{2}$-type has a relatively high energy, suggesting that orbital ordering is not responsible for the magnetic structure [12].

The photoemission study of $\mathrm{PrNiO}_{3}$ has shown that the charge-transfer energy $\Delta$ of $\mathrm{PrNiO}_{3}$ is $\sim 1 \mathrm{eV}$ and that the ground state is a mixture of the $d^{7}$ and $d^{8} \underline{L}$ configurations, where $\underline{L}$ denotes a hole at the oxygen $2 p$ orbitals. Since the ground state has a large amount of oxygen $2 p$ holes, it is also possible to describe it starting from the $d^{8} \underline{L}$ state. In this picture, the system can be viewed as a self-doped Mott insulator and the antiferromagnetic and insulating state in $\mathrm{PrNiO}_{3}$ and $\mathrm{NdNiO}_{3}$ may be interpreted as a spin and charge ordered state in the selfdoped Mott insulator. Indeed, our calculations confirmed the existence of such ordered states which are consistent with the neutron diffraction measurement. They are illustrated in Fig. 1. In the state shown in Fig. 11(a), half of the oxygen sites have more holes than the other half. The excess holes located at the oxygen sites cause the ferromagnetic coupling between the neighboring two $\mathrm{Ni}$ spins. Therefore, the up-up-down-down stacking of the ferromagnetic planes along the $(1,1,1)$-direction is realized without orbital ordering. On the other hand, all the $\mathrm{Ni}$ sites have the same number of $3 d$ electrons. Let us denote this state as an oxygen-site charge-ordered (OCO) state. In the state shown in Fig. 11(b), while all of the oxygen sites are occupied by the same amount of holes, half of the Ni sites have more $3 d$ electrons than the other half. This state can be called a metal-site charge-ordered (MCO) state.

In Fig. 2(a), the energies of the spin and charge ordered states relative to the FM and metallic state are plotted as functions of the charge-transfer energy $\Delta$ for the cubic perovskite lattice. For $\Delta \leq 1 \mathrm{eV}$, the OCO and MCO states exist as stable solutions. The OCO state is lower in energy than the MCO state for $-5 \mathrm{eV} \leq \Delta \leq$ $1 \mathrm{eV}$. At $\Delta=-7 \mathrm{eV}$, the OCO and MCO states are almost degenerate in energy. This result indicates that, as the charge-transfer energy $\Delta$ decreases, the MCO state becomes favored compared to the OCO state. In Fig. 2(b), the energies of the $\mathrm{OCO}$ and $\mathrm{MCO}$ states relative to the FM state are plotted for the perovskite lattice with the orthorhombic distortion which is due to the tilting of the $\mathrm{NiO}_{6}$ octahedra. Here, the tilting angle is $15^{\circ}$ which is a typical value found in $R \mathrm{NiO}_{3}$ [10]. At $\Delta=-7 \mathrm{eV}$, the MCO state is slightly lower in energy than the OCO state, indicating that the orthorhombic distortion or the $\mathrm{GdFeO}_{3}$-type distortion favors the MCO state. However, for $\Delta \geq-5 \mathrm{eV}$, the OCO state has lower energy than the MCO state even with the substantial distortion. Since, in $\mathrm{PrNiO}_{3}$ and $\mathrm{NdNiO}_{3}$, every $\mathrm{Ni}$ site has the same magnitude of the magnetic moment [11, it is reasonable to attribute the antiferromagnetic and insulating state in $\mathrm{PrNiO}_{3}$ and $\mathrm{NdNiO}_{3}$ to the $\mathrm{OCO}$ state. In the present model calculation without lattice distortions, the OCO state is higher in energy than the FM and metallic state for realistic $\Delta$. However, since the charge ordering at the oxygen sites is expected to strongly couple with a lattice relaxation, a structural modulation may stabilize the OCO state as discussed in the following paragraphs.

The number of $3 d$ holes $N_{d}^{h}$ and spin $S_{d}$ at the Ni sites are plotted as functions of $\Delta$ in Fig. 3(a). In the OCO state, $N_{d}^{h}$ is uniform at all the $\mathrm{Ni}$ sites. As $\Delta$ decreases, $N_{d}^{h}$ becomes smaller because the transfer of holes from the oxygen sites to the $\mathrm{Ni}$ sites increases. In these solutions, $N_{d}^{h}$ are approximately two and the population of the $d_{x^{2}-y^{2}}$ orbital is the same as that of the $d_{3 z^{2}-r^{2}}$ orbital, indicating that, in the OCO state, Ni is essentially +2 and the orbital degeneracy is lifted. On the other hand, $N_{d}^{h}$ are 2.00 and 2.28 in the MCO state for $\Delta=1$ $\mathrm{eV}$. The Ni sites with $N_{d}^{h}$ of 2.00 have the spin $S_{d}$ of 0.80 and are $\mathrm{Ni}^{2+}$ as those in the OCO state. The $\mathrm{Ni}$ sites with $N_{d}^{h}$ of 2.28 have no spin and is well described by the $d^{8} \underline{L}^{2}$ state which can hybridize with the low-spin $d^{6}$ state. In this sense, the $\mathrm{Ni}$ sites can be viewed as $\mathrm{Ni}^{4+}$ like (low-spin $d^{6}$ ) sites. Therefore, the MCO state is a kind of charge disproportionated state in which two $\mathrm{Ni}^{3+}$ sites are turned into the $\mathrm{Ni}^{2+}$-like and $\mathrm{Ni}^{4+}$-like sites as pointed out by Solovyev et al. based on $\mathrm{LDA}+U$ calculation [15]. An antiferromagnetic ordering of magnetic $\mathrm{Ni}^{2+}$-like sites (see Fig. 1(b)) is also consistent with the neutron diffraction results $[11$. As $\Delta$ decreases, the difference of $N_{d}^{h}$ between the $\mathrm{Ni}^{2+}$-like and $\mathrm{Ni}^{4+}$-like sites becomes smaller in the MCO state. The difference almost disappears at $\Delta=-7 \mathrm{eV}$, where the MCO state is almost degenerate in energy with the OCO state. Here, it is interesting to note that the charge disproportionation of $2 \mathrm{Fe}^{4+} \rightarrow \mathrm{Fe}^{3+}+\mathrm{Fe}^{5+}$ has been observed in $\mathrm{CaFeO}_{3}$ 
[9. which has highly negative charge-transfer energy $\Delta$ [5]. The number of $2 p$ holes $N_{p}^{h}$ at the oxygen sites are plotted as functions of $\Delta$ in Fig. $3($ (b). The OCO state has the hole-rich and hole-poor oxygen sites. For $\Delta$ of 1 $\mathrm{eV}, N_{p}^{h}$ is $\sim 0.33$ at the hole-rich oxygen sites and is $\sim$ 0.22 at the hole-poor oxygen sites. On the other hand, in the MCO state, $N_{p}^{h}$ is uniform at all the oxygen sites.

Recently, Medarde et al. have observed strong ${ }^{16} \mathrm{O}-$ ${ }^{18} \mathrm{O}$ isotope effect on the metal-insulator transition of $R \mathrm{NiO}_{3}$, [16] indicating that the electron-lattice coupling is important in $R \mathrm{NiO}_{3}$. Very recently, Alonso et al. performed neutron diffraction studies of $\mathrm{YNiO}_{3}$ and found the breathing-type distortion which may be an indica-

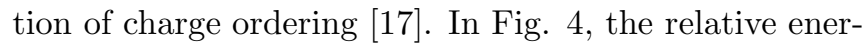
gies of the OCO and MCO states compared to the ferromagnetic state are plotted for $\Delta$ of $-1 \mathrm{eV}$ as functions of the various lattice distortions with which the charge orderings are expected to couple. The MCO state becomes stable with the breathing-type lattice distortion as shown in Fig. 1 (a). Here, $\delta_{\mathrm{O}}$ is the shift of the oxygen ions which gives the breathing-type distortion. The MCO state becomes the lowest in energy for rather small distortion, indicating that the MCO state coupled with the breathing-type distortion is relevant for $\mathrm{YNiO}_{3}$. The OCO state can be stabilized with the modulation of the bond length which is a consequence of the shift of the $\mathrm{Ni}$ ions as shown in Fig. 4(b). Here, the shifts of the $\mathrm{Ni}$ ion are along the $(1,1,1)$ direction and are given by $\left(\delta_{\mathrm{Ni}}, \delta_{\mathrm{Ni}}, \delta_{\mathrm{Ni}}\right)$ and $\left(-\delta_{\mathrm{Ni}},-\delta_{\mathrm{Ni}},-\delta_{\mathrm{Ni}}\right)$. Consequently, the Ni-O bond length for the FM coupling becomes shorter and that for the AFM coupling becomes longer. Fig. 1( (c) shows that the OCO state is also stabilized by the modulation of the bond angle which is derived from the tilting of the octahedra and the shift of the oxygen ions. In this model distortion, the Ni-O-Ni bond angle for the FM coupling is $180^{\circ}$ and that for the AFM coupling is smaller than $180^{\circ}$. In Fig. 田(c), the relative energy is plotted as a function of the smaller Ni-O-Ni band angle. Although these distortions can stabilize the OCO state, we need unreasonably large modulations in order to make the OCO state lower in energy than the FM state. We need more experimental and theoretical investigations to identify the lattice distortion realized in $\mathrm{PrNiO}_{3}$ and $\mathrm{NdNiO}_{3}$.

In conclusion, we have studied spin and charge ordered states in self-doped Mott insulators with small or negative charge-transfer energy. It was found that two types of charge ordered states are possible: the OCO state with charge ordering at the oxygen sites and the MCO state with charge ordering at the transition-metal sites. The present HF calculation without distortion has shown that the OCO state has lower energy than the MCO state for moderately small $\Delta$ and that the OCO and MCO states are almost degenerate for highly negative $\Delta$. Since, in $\mathrm{PrNiO}_{3}$ and $\mathrm{NdNiO}_{3}$, every $\mathrm{Ni}$ site has the same magnitude of the magnetic moment [11], the antiferromag- netic and insulating state in $\mathrm{PrNiO}_{3}$ and $\mathrm{NdNiO}_{3}$ can be attributed to the OCO state of the self-doped Mott insulators. The OCO state in the self-doped Mott insulators is novel in that, even without explicit doping, the spin ordering at the transition-metal sites and the charge ordering at the oxygen sites coexist and couple with each other just like the spin and charge ordered states in the doped Mott insulators. On the other hand, for $\mathrm{YNiO}_{3}$, the strong breathing-type distortion stabilizes the MCO state. Here, it is interesting to note that $\Delta$ of $\mathrm{CaFeO}_{3}$ is highly negative and can have the $\mathrm{MCO}$ state even without the strong beathing-type distortion. The charge disproportionated state observed in $\mathrm{CaFeO}_{3}$ may be regarded as a kind of MCO state in the selfdoped Mott insulators. In $R \mathrm{NiO}_{3}$ and $\mathrm{CaFeO}_{3}$, the homogeneous state corresponds to an orbitally degenerate state $\left(t_{2 g}^{6} e_{g}^{1}\right.$ for $\mathrm{Ni}^{3+}$ and $t_{2 g}^{3} e_{g}^{1}$ for $\left.\mathrm{Fe}^{4+}\right)$. The charge disproportionation observed in $\mathrm{CaFeO}_{3}$ and the $\mathrm{OCO}$ and MCO states for $R \mathrm{NiO}_{3}$ may be another way to get rid of this orbital degeneracy besides the usual cooperative Jahn-Teller (or orbital) ordering.

The authors would like to thank M. Medarde, J. Rodriguez-Carvajal, J. L. Garcia-Muños, J. Matsuno, A. Fujimori, I. Solovyev and J. B. Goodenough for useful discussions. This work was supported by the Nederlands Organization for Fundamental Research of Matter (FOM) and by the European Commission TRM network on Oxide Spin Electronics (OXSEN).

[1] J. Zaanen, G. A. Sawatzky, and J. W. Allen, Phys. Rev. Lett. 55, 418 (1985); S. Hüfner, Z. Phys. B 61, 135 (1985).

[2] T. Mizokawa et al., Phys. Rev. Lett. 67, 1638 (1991); 70, 1565(E) (1993); Phys. Rev. B 49, 7193 (1994); S. Nimkar et al., Phys. Rev. B 48, 7355 (1993).

[3] T. Mizokawa et al., Phys. Rev. B. 57, 9550 (1998).

[4] S. R. Barman, A. Chainani, and D. D. Sarma, Phys. Rev. B 49, 8475 (1994); T. Mizokawa et al., Phys. Rev. B. 52, 13865 (1995).

[5] A. E. Bocquet et al., Phys. Rev. B. 45, 1561 (1992); J. Matsuno et al., to be published in Phys. Rev. B.

[6] D. I. Khomskii, Lithuanian Journal of Physics 37, 65 (1997).

[7] M. A. Korotin et al., Phys. Rev. Lett. 80, 4305 (1998).

[8] C. H. Chen, S.-W. Cheong, and A. S. Cooper, Phys. Rev. Lett. 71, 2461 (1993); V. Sachan et al., Phys. Rev. B 51, 12742 (1993).

[9] M. Takano et al., J. Solid State Chem. 39, 75 (1981).

[10] J. B. Torrance et al., Phys. Rev. B. 45, 8209 (1992); M. Medarde et al., Phys. Rev. B. 46, 14975 (1992).

[11] J. L. Garcia-Muños, J. Rodriguez-Carvajal and P. Lacorre, Phys. Rev. B. 45, 8209 (1994); J. RodriguezCarvajal et al., Phys. Rev. B. 57, 456 (1998). 
[12] T. Mizokawa and A. Fujimori, Phys. Rev. B 51, 12880 (1995); T. Mizokawa and A. Fujimori, Phys. Rev. B 54, 5368 (1996).

[13] J. Kanamori, Prog. Theor. Phys. 30, 275 (1963).

[14] W. A. Harrison, Electronic structure and the Properties of Solids (Dover, New York, 1989).

[15] I. V. Solovyev et al., unpublished.

[16] M. Medarde et al., Phys. Rev. Lett. 80, 2397 (1998).

[17] J. A. Alonso et al., Phys. Rev. Lett. 82, 3871 (1999).

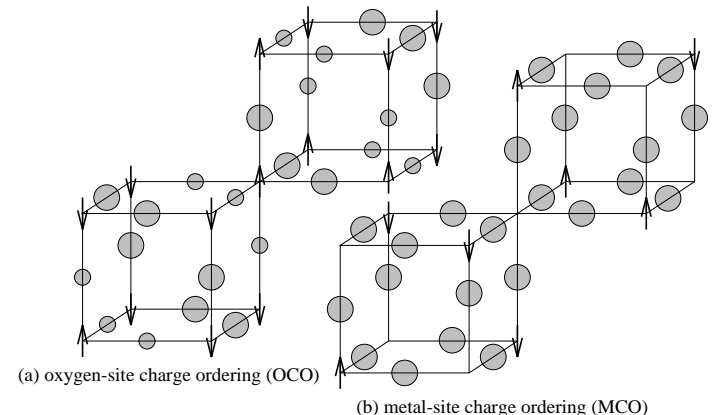

FIG. 1. Schematic spin and charge orderings (a) for the oxygen-site charge-ordered (OCO) and (b) for metal-site charge-ordered (MCO) states. The arrows and the circles indicate the $\mathrm{Ni}$ spins and the oxygen $2 p$ holes, respectively. The larger and smaller circles are for the hole-rich and hole-poor oxygen sites, respectively.

(a) $\mathrm{RNiO}_{3}$ without tilting
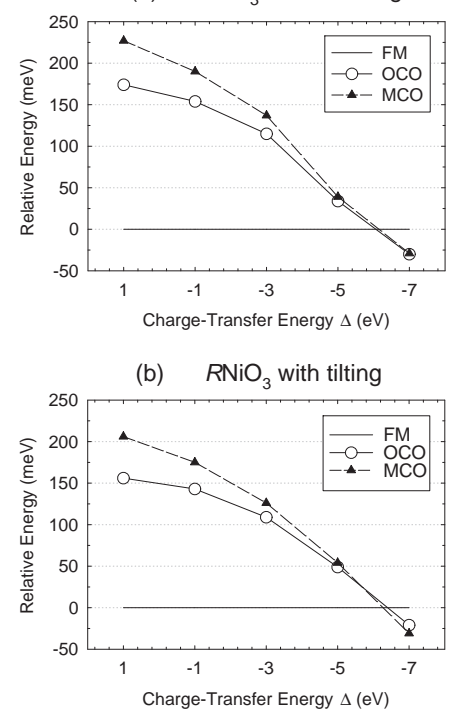

FIG. 2. Energies of the $\mathrm{OCO}$ and $\mathrm{MCO}$ states relative to the ferromagnetic and metallic state as functions of the charge-transfer energy $\Delta$ (a) for the cubic perovskite lattice and (b) for the perovskite-lattice with the orthorhombic distortion.
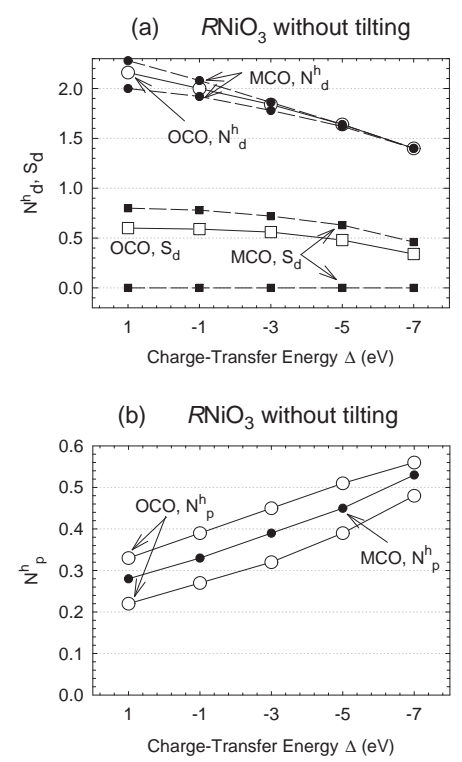

FIG. 3. (a) Number of $3 d$ holes $N_{d}^{h}$ and spin $S_{d}$ at the Ni sites as functions of the charge-transfer energy $\Delta$. (b) Number of $2 p$ holes $N_{p}^{h}$ at the oxygen sites as functions of the charge-transfer energy $\Delta$.
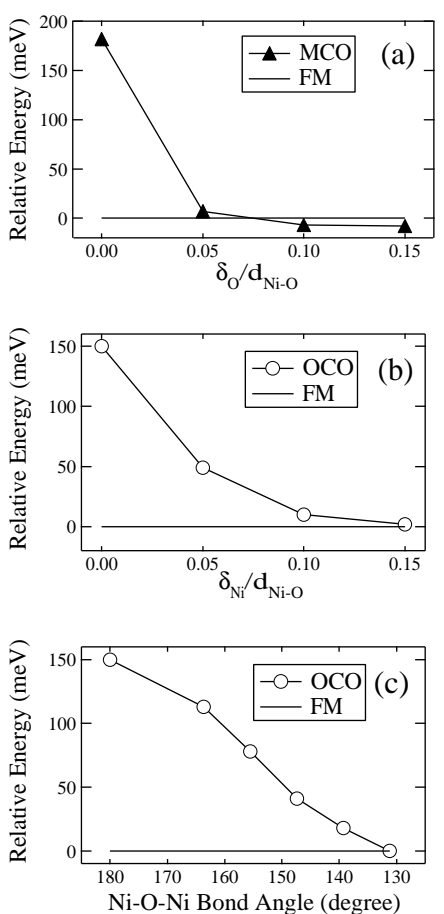

FIG. 4. Energies of the OCO and MCO states relative to the FM state as functions of (a) the breathing-type distortion given by the shift of the oxygen ions, (b) the modulation of the bond length driven by the shift of the Ni ions, and (c) the modulation of the bond angle driven by the shift of the oxygen ions. $\delta_{\mathrm{O}}$ and $\delta_{\mathrm{Ni}}$ are the shifts of the oxygen and Ni ions. $d_{\mathrm{Ni}-\mathrm{O}}$ is the Ni-O bond length for the undistorted lattice. 